\title{
Against Selective Realism(s)
}

\author{
D. Tulodziecki ${ }^{*}$ \\ Draft/October 2016
}

\begin{abstract}
It has recently been suggested (for example, Lyons 2006) that realist responses to historical cases featured in pessimistic meta-inductions are not as successful as previously thought. In response, selective realists have updated the basic divide et impera strategy specifically to take such cases into account, and to argue, on this basis, that more modern realist accounts are immune to the historical challenge (cf. Vickers 2013). Using a case-study - that of the 19th century zymotic theory of disease - I argue that these updated proposals fail, and that even the most sophisticated recent realist accounts are just as vulnerable as their predecessors.
\end{abstract}

\section{Introduction}

The pessimistic meta-induction (PMI) targets the realist's claim that a theory's (approximate) truth is the best explanation for its success. It attempts to do so by undercutting the alleged connection between truth and success by arguing that highly successful, yet wildly false, theories are typical of the history of science $\|^{1}$ There have been a number of prominent realist responses to the PMI, most notably those of Worrall (1989), Kitcher (1993), and Psillos (1999). All of these responses try to rehabilitate the connection between a theory's (approximate) truth and its success by attempting to show that there is some kind of continuity between earlier and later theories. One of the most widely discussed proposals has been Psillos's divide et impera strategy.

*tulodziecki@purdue.edu

${ }^{\dagger}$ Department of Philosophy, Purdue University, West Lafayette, IN

${ }^{1}$ For a recent and new take on the PMI, see Frost-Arnold (2014). 
Psillos argues (1999, Chapter 5), first, that realists ought to make the notion of a theory's success more stringent so as to include use-novel predictions, and, second, that realists face trouble only if it can be shown that those elements of a theory that "really fuel" that theory's genuine success are rejected and turn out to be completely false. It has recently been suggested for example, by Lyons (2006) -, however, that Psillos's strategy is not as successful as previously thought. Lyons tests Psillos's move against a number of historical cases, and concludes that this "form of realism remains threatened by the historical argument that prompted it" (537). In response to Lyons, recent realists such as Vickers (2013) have argued that, once the selective realist strategy is updated appropriately, more modern realist accounts can, in fact, meet the challenge that Lyons has set.

In this paper, I argue that even recent, sophisticated realist accounts such as that of Vickers fail to be immune to the historical challenge and are just as vulnerable as their predecessors. I make my point by providing an example of such a case - that of the 19th century zymotic theory of disease, predecessor to the germ theory - and by carefully showing that this theory was highly successful in the realist's sense of 'genuine success'. I explain in detail what elements of the theory were responsible for its successes, by providing derivations of its predictions and the theoretical posits involved in making these predictions, and then show that the elements responsible for its success and that "really fueled" the relevant derivations were discarded in later theories and turned out to be completely false.

I will proceed as follows: In Section 2, I provide an overview of the zymotic theory of disease; in Section 3, I discuss its successes. Section 4 deals with the updated realist challenge and Section 5 is concerned with a derivation of the zymotic predictions and those posits of the zymotic theory that brought them about. In Section 6, I show how the updated realist challenge can be met, before concluding, in Section 7, that even the most sophisticated recent realist accounts are in trouble just as much as their predecessors.

\section{The Zymotic Theory}

The zymotic theory was the most sophisticated version of the miasma theory and dominated British disease theory from the 1840s to the 1870s (although it is frequently referenced well into the early 1900s). It sought to explain diseases in terms of complex interactions between miasmas and so-called 
zymotic materials. Miasma was the result of rotting organic matter produced by decomposition processes. It would be dispensed in the air which, in turn, would act, via zymotic principles, on individual constitutions, causing one of several diseases (cholera, yellow fever, typhus, etc.), depending on a number of more specific factors. Some of these were thought to be extraneous, such as weather, climate, and humidity, and would affect the nature of the miasmas themselves; others were related directly to the potential victims and thought to render them more or less susceptible to disease. Lastly, there were a variety of local conditions that could exacerbate the course and severity of the disease, such as overcrowding and bad ventilation.

The term 'zymotic' (from the Greek for fermentation) goes back to William Farr (1807-1883), Statistical Superintendent of the General Register Office from 1842 to 1879. Farr coined this term to indicate that disease processes "are of a chemical nature, and analogous to fermentation; by which they are moreover to a certain extent explained" $(1842,201)$, yet not identical to vinous fermentation. Since decomposition figured heavily in the various accounts of disease causation, disease theorists drew heavily on contemporary chemical theories, such as those of Liebig, who had both a comprehensive system for explaining the various morbid processes of decomposition, putrefaction, and fermentation, but also his own specific zymotic pathology. Chemical theories like those of Liebig were well suited to explaining diseases, because they explained the interaction between living and non-living things, such as human bodies and the environment, and they did this on a molecular basis. Moreover, Liebig's chemical theories were popular, highly respectable, and they had already had great successes, and so the zymotic theory may be seen as drawing on some of the most successful science at the time.

Liebig and Farr held a so-called contact theory of decomposition. According to this, diseases occur as a result of introducing into the body (through inhalation or direct contact) various zymotic principles. These were thought to be "organic matter in a state of pathological transformation" (Farr 1842, 202). This would be absorbed by the blood, and, through the transformation, zymotic diseases had "the property of communicating their action [i.e. decomposition], and effecting analogous transformations in other bodies" (ibid.). The zymotic principles were the 'exciters' of the various diseases and "in the blood corresponding bodies exist, which are destroyed, and by the transformation of which the exciters are generated or reproduced" (ibid., 199). In short, pre-existing stuff in a victim's blood catches the process of decomposition and communicates this state to other particles of blood (which, 
in turn, would transmit it to various body parts), until it ran out of susceptible particles to contaminate. The underlying idea of the contact theory was that zymotic matter was like ferment, a volatile chemical substance that could transfer its volatility to other materials. So, just as ferment produced fermentation, zymotic material produced disease (zymosis).

Two things about the zymotic theory are worth stressing: first, zymotic material was not a specific substance; according to the zymotic theory, the disease was not the (presence of) zymotic materials, but the zymotic processes of transformation. Second, the zymotic account was purely chemical, and Liebig (and others) explicitly rejected the view that zymotic materials were living organisms.

\section{Successes of the Zymotic Theory}

The zymotic theory was highly successful with respect to a number of phenomena. Specifically, it was successful both in terms of explanation and prediction. Among its explanatory successes were explanations of well-known disease phenomena, such as the fact that diseases were known to be seasonal, and often tied to particular regions (such as marshy ones) or particular locations (barracks, prisons, etc.). Similarly, it was well known that sickness and mortality rates in poor, crowded urban centres were worse than in their less poor and crowded counterparts, and that, in turn, those parts were affected worse than rural areas. It could also explain a number of facts tied to epidemics, such as why epidemics began, took the course they did, and then subsided, yet often came back several years later. It could account for the fact that epidemic diseases often moved around when there was no known contact with previous victims, why quarantines were ineffective for some diseases (such as cholera), why only some, but not all people were affected by a given disease, and, lastly, why certain diseases were endemic.

The zymotic theory could explain all of these phenomena through its claims that decomposing material produced miasmas. Diseases peaked when conditions for putrefaction were particularly favourable: this was the reason why certain diseases were particularly bad during periods of high temperature and in certain geographical regions (for example, the many fevers in Africa), why urban centres were much more affected than rural areas, and why even specific locations in otherwise more or less healthy areas could be struck (sewage, refuse, and general 'filth' would sit around in badly ventilated areas). More- 
over, since zymotic material interacted with the blood, the zymotic theory could provide an account for individual disease susceptibility, and explain why certain diseases were contracted only once (once the relevant material in a victim's blood had been 'converted', the person became immune).

While this degree of explanatory success is quite impressive, as we have seen, however, realists tend to think that, in addition, genuine success also requires a theory to make use-novel predictions, i.e. predictions that did not play a role in the theory's original formulation. Here, the zymotic theory also delivers. It predicted, for example,

(i) that the air in areas with higher disease incidence ought to be worse than the air in healthier areas; more specifically, that it should contain more decomposing organic material,

(ii) a number of different disease incidence patters, based on the prevalence of decomposing and putrefying materials (in conjunction with facts about ventilation), including

(a) relationships between disease prevalence and season, temperature, rainfall, wind, and so on,

(b) a relationship between disease incidence and population density,

(c) a relationship between disease incidence and elevation,

(iii) facts about the course and duration of various epidemics,

(iv) facts about the relation between mortality rates and different occupations, and, lastly,

(v) facts about relationships between mortality from various diseases and age.

Thus, the zymotic theory had successes on both explanatory and predictive levels. Before showing (in Section 6) that these successes were, in fact, due to a number of essential working posits that did not get retained in successor theories and that turned out to be completely false, however, let us be clear about what exactly the new selective realist challenge amounts to. 


\section{The New Selective Realist Challenge}

In response to the pessimistic meta-induction, besides making the notion of success more stringent, selective realists have suggested that we ought to focus only on those parts of past theories that were, in fact, responsible for their genuine success. Prominent approaches include Worrall's structural realism, Kitcher's distinction between working and presuppositional posits, and Psillos's divide et impera strategy. And, while this line of response has also been popular among more recent realists, such as Harker (2013), Peters (2014), Saatsi (2005), and Vickers (2013), they also acknowledge the shortcomings of the traditional responses. In this vein, Vickers, for example, argues that the basic divide et impera strategy needs to be updated, since (i) first, there now are cases of successful theories that did make novel predictions but that, nevertheless, turned out to be completely false (cf. Lyons 2006), and (ii) second, "the divide et impera position needs significant refinement, especially concerning the crucial concepts scientific success and responsible for on which so much weight is placed" $(2013,190)$.

Thus, Vickers agrees with his predecessors that a given historical case poses a problem only "if posits that 'really fuel the derivation' [of a novel prediction] turn out to be definitely not approximately true" (194), but he thinks more light needs to be shed on what it means for a posit to "really fuel a derivation". To this effect, Vickers proposes a distinction between derivationexternal and derivation-internal posits. The former are those that "merely influenced the thinking of scientists" (198), but, since they only guide scientists and are not part of the relevant derivation, they are not eligible for realist commitment. The latter are those "posits [that] were actually involved in the derivation of that prediction" (198). However, Vickers argues, derivation-internal posits are not "necessarily the 'working posits' since any individual posit might 'contain within it' some other posit that is the real working part" (198). In other words, a posit might contain a 'weaker' posit that is sufficient for the prediction, such as the posit "the passengers are $50 \mathrm{~kg}$ too heavy" containing within it the weaker posit "the passengers are too heavy" (198.; originally due to Saatsi 2005, 532).

Thus, contrary to previous realists - especially Psillos, according to whom scientists' judgements play an important role in determining what fuelled a derivation - Vickers thinks that realists ought to commit themselves only to posits that do logical work, and moreover, to their weakest possible versions (i.e. if one weakened the posits any more, one could no longer derive the 
prediction). Thus, Vickers makes a distinction between actual, historical derivations, and (possibly much weaker) logical, epistemic derivations (cf. Peters 2014, 386). And, while Vickers believes there are now cases showing that there are theories that contain working posits that turned out to be false, he does not think that there are cases showing that there are essential parts of derivation-internal posits that turned out to be false. Coming up with such cases, then, is the new and updated selective realist challenge.

\section{$5 \quad$ Zymotic Predictions}

In order to show that the zymotic theory can rise to it, let's look at some of its predictions in more detail, starting with its predictions concerning air quality. Here, the zymotic theory predicts, (i) first, that air quality ought to be proportional to disease incidence, so that the 'right' locations should have good and bad air, respectively, and (ii) second that differences in air quality ought to be related to decomposition and ventilation. A number of mid-19th century chemists tried to test these predictions; however, for brevity's sake, I will restrict my focus to a small subset of the experiments of Robert Angus Smith (1817-1884), a contemporary of Farr's, and often cited by the latter. ${ }^{2}$

Smith began by collecting indoor condensation liquid from crowded rooms and compared it to fresh rainwater, finding that the indoor liquid, but not the rainwater, had a strong perspiration smell, and, "on standing it formed a glutinous mass in which the microscope revealed "Confervae", "greenish globules", "various species of Volvox" [a type of algae], and "monades many times smaller"" (ibid., 219-220; Smith, 1848, 18). While this was not a strong result by itself, Smith believed that it at least showed that the the indoor liquid contained organic material on which the Volvox and the monades were feeding (ibid., 220). Further, upon burning the liquid's residue, he obtained the smell of ammonia, which was significant, since ammonia was tied to the last stage in decomposition. At the larger scale of towns, these results were thought to be exacerbated, not just because of the various exhalations of living bodies, but, in addition, those of animal refuse and fuel combustion (Smith, 1848).

Smith also tried to measure air quality more directly. He washed air samples by changing the air in a bottle containing distilled water, shaking the

\footnotetext{
${ }^{2}$ Smith was not a particularly distinguished scientist (Eyler 1980, 216), but is, for this reason, quite representative of a number of people and their work.
} 
bottle after each change of air (with up to 200 changes), and then performed a chemical analysis on the water, determining its amount of organic material. He found these results to confirm those previously obtained from the experiments above (Smith 1859, 218-225; cf. also Eyler, 220).

Most telling, however, were experiments in which he tried to show that there was organic material in vapours given off by putrefying meat, blood (due to the strong smell, he only performed a limited number of these), and cesspools. He proceeded to compare these samples to the air in a number of different locations - everything from different areas of Manchester, to "closely packed railway carriages[s]" $(1859,221)$, to the air in bedrooms before and after someone had slept in them, to the occasion on which a "strong smell of a sewer entered my laboratory" (221), the fronts and backs of various houses, alleys, and so on. Smith obtained rather a large variation in air quality, and, specifically, concluded that by inhaling putrid air from decomposing animal matter (such as decomposing mutton), "we might be inhaling 9000 times more of some organic substance or other than we should be doing by inhaling the purest air" $(1859,222)$. These figures include the vapours given off by the putrefying meat, but, without these, he concluded that the difference among different areas of town is about 22 times, and, within industrial areas, ranges from about 9 to 22 times, while over the Atlantic Ocean and the Highlands, the air was clean (223; cf. also Eyler 220-221). Most importantly, however, as Eyler points out, the ranges Smith found were in proportion "to the range of the crude death rates for the districts of Manchester" (221): in the districts with the highest death rates, the air had most organic material in it, and the ones with the lowest death rate had the cleanest air. Smith concludes that "[t]hese differences ... enable us to account for the number which represent the deaths of the various districts" $(1859,223)$.

He also performed an investigation for the mining commission, during which he observed people in air-tight lead chambers, recording their pulse, respiration, and so on. He systematically measured the carbonic acid concentration inside the chamber, and concluded that carbonic acid was quite harmful, that it "almost always comes in bad company" (Eyler, 222), and then proceeded to use carbonic acid concentrations to test how well or badly ventilated a given place was.

However, Angus Smith's experiments are not the only ones speaking in favour of the zymotic theory. As we have already seen, the zymotic theory also predicted a number of other relationships, such as those between disease incidence and population density and between disease incidence and distance 
from sources of decomposition. Both of the latter were confirmed by Farr. First, Farr showed that "the mortality of town districts has a certain relationship to their density" (207). Based on his analyses of the data sets of a number of Annual Reports of the General Register Office, Farr came up with a law on whose basis he made a number of precise predictions. When he then proceeded to compare the calculated, expected mortality to that actually observed in the 30 London statistical districts, he found the results agreeing "very directly with the results of direct observation" (ibid.; due to space constraints, I won't discuss this result in any detail, but, for a quick flavour of the kind of law Farr put forth, see figure 1 (ibid.)).

Figure 1: Farr's Density Equation

Given the mortality of St. James's district $=\cdot 02145$; the density of the population 145059 to a square mile; the density of St. George's, Hanover-square, 39018 to a square mile, what was the mortality of St. George's, Hanover-square?

Here $\sqrt[6]{\frac{d}{d} m^{\prime}}=m$; and substituting the figures in the formula, the result agrees very exactly with the results of direct observation $\sqrt[6]{\frac{39018}{145059}} \times \cdot 02145=\cdot 0172$, the mortality of St. George, Hanover-square, the mortality observed having been $\cdot 0171$.

The mortality of the city of London deduced from the mortality of St. George, Hanoversquare, and the densities of the two districts is

$$
\sqrt[6]{\frac{d^{\prime}}{d} m}=m^{\prime} ; \text { or } \sqrt[6]{\frac{94488}{39018}} \times \cdot 01707=\cdot 0199 .
$$

The density of a district is deducible from the same formula. It may be expressed, however, differently; namely, by the number of square yards to a person, and denoted by $y^{\prime}$ in the district where the number of square yards to a person is greatest: then

$\frac{m}{m^{\prime}}=\frac{\sqrt[6]{y}}{\sqrt[6]{y^{\prime}}} ;$ and $\left(\frac{m}{m^{\prime}}\right)^{6}=\frac{y}{y^{\prime}} ;$ consequently, $y=\left(\frac{m}{m^{\prime}}\right)^{6} y^{\prime}=$ the number of square yards to

a person in the least dense district.

His crowning achievement, however, was his elevation law, relating cholera mortality to soil elevation. This relation relation followed straightforwardly from those parts of the zymotic theory having to do with the dilution of miasma in the atmosphere. Here, what Farr did was to capture the exact relation between the decline of cholera and increased soil elevation in the form of the following equation: $c=c^{\prime} \times\left(e^{\prime}+a\right) /(e+a)(e$ is the elevation above the Thames high water mark, $c$ the average cholera mortality rate at that elevation, and $e^{\prime}$ and $c^{\prime}$ the elevation and mortality at a higher elevation, $a$ is a constant; 1852, lxiii). Farr then calculated the expected series according to the formula, compared it to the actual series recorded in London, and found remarkable agreement (1852, lxiii). Seeking further confirmation, he 
then proceeded to "submit the principle to another test, by comparing the elevation and the mortality from cholera of each sub-district", and found that this "entirely confirms the announced law" (xv-xvi). Lastly, Farr's predictions were also confirmed by others in different regions. For example, "William Duncan, Medical Officer of Health for Liverpool, wrote that when he grouped the districts of his city by elevation as Farr had done, that cholera mortality in the last epidemic obeyed Farr's elevation law for Liverpool as well" (Eyler, 1979, 228).

\section{Meeting the New Realist Challenge}

Note that all of the above predictions were use-novel: they could not have played a role in the construction of the zymotic theory, since they were not even formulated by then, and, in Farr's case, the data on which his laws were based did not even exist. As such, the case of the zymotic theory meets the realists' criteria for 'genuine success'.

However, as we have seen, Vickers does not think that this is enough; in addition, he requires that it be shown that the derivation of these predictions involved ineliminable parts of derivation-internal posits that cannot be weakened. Here, I want to argue that the zymotic theory can meet this challenge, too.

The first question is what the zymotic theory's relevant posits are. Crucial to the derivations of the above predictions are the following three:

1. Organic matter given off by putrefying material is the exciting cause of diseases.

2. This organic matter is suspended in the air (i.e. there are miasmas).

3. This, in turn, is transmitted through the air.

Clearly, all of the above posits are derivation-internal, not derivationexternal: they did not just guide practitioners' thinking, but they were all crucially involved in making the various predictions. They were essential in the air quality predictions confirmed by Angus Smith: if any of the above posits are taken away, the entire prediction disappears. While the second and third are directly about quality, the first is also necessary: without specific reference to (sources of) decomposition, we lose the geographical and 
other location patterns, and, as a result, the entire prediction about air differences. Thus, all of these posits are clearly doing work in the production of the prediction. Similarly for Farr's predictions about density and elevation. Both putrefying material as a source of miasma and air as a medium of its transmission are crucial, since, taking either one away makes the prediction disappear. Thus, it is clear that the above posits are doing work.

However, as we have seen, according to Vickers, this is not enough - it also needs to be the case that they don't entail weaker versions of themselves, on the basis of which the prediction still goes through. Examining our three posits, we see that this, too, is the case. Ironically, the fact that the zymotists knew relatively little about the chemical make-up of the alleged miasmas is to their advantage here, since, absent any specific information about the make-up of the allegedly responsible organic matter, these posits are already as weak as they can get: they effectively state that whatever is given off during decomposition is transmitted through the air and involved in causing diseases. Weakening them any more would take away either the decomposing sources, or air as a medium, and, as we have already seen, both of these are necessary in order to make the predictions in the first place. Thus, since these posits cannot be weakened, they ought to count as essential parts of derivation-internal posits, according to Vickers. Moreover, they did not get retained in any way, shape, or form, in the germ theory: decomposition is not responsible for disease, neither is disease material dispensed in the air, neither is the air a medium for disease transmission. But, if that is right, according to Vickers, and by his own admission, we ought to have been realists about miasmas 3

So much for miasmas, but what about zymes? Further zymotic posits we might add to the miasmatic ones above are:

1. Diseases are a type of decomposition,

2. Disease processes are analogous to processes of fermentation (which is a type of decomposition),

3. Zymotic material acts like a ferment on pre-existing stuff in a victim's blood.

\footnotetext{
${ }^{3}$ Note that this poses a problem not just for Vickers, but for selective realists more generally.
} 
Now, clearly, none of these posits are necessary for the predictions we have seen, since it was possible to provide derivations of these predictions without appealing to any of the zymotic posits. Thus, obviously, they are non-essential. However, I want to stress at this point that, even if the zymotic posits fail to be essential, note that the miasmatic posits clearly are, and that this is already enough of a problem for selective realists. After all, selective realists face trouble if essential parts of derivation-internal posits turn out to have been completely false, and the miasmatic posits fit that bill. But, of course, it is not a requirement that every (rejected) posit be essential (which is obviously not right). Thus, showing that the miasmatic posits are essential and were rejected suffices to get the realist into trouble.

More interesting, however, is the fact that even though the zymotic posits might not have been essential for making predictions, they play a different, and perhaps equally important role in the zymotic theory: that of providing unifying explanations ${ }^{4}$ More specifically, among other things, the zymotic posits explain why there is a link between decomposition and disease (they are essentially variations of each other), why diseases vary with season and temperature (it was well known that fermentation processes are temperaturesensitive), why certain diseases only occurred once (victims' blood would run out of material to ferment), why certain diseases were childhood diseases (children's blood was different from adults'), and why epidemics began (existence of sufficiently virulent zymotic material) and ended (lack of new victims with the appropriate blood). Lastly, it had been clear for some time that disease material needed to replicate itself somehow, and fermentation processes offered an explanation of how this was possible. The various zymotic posits above can make sense of all of these at once. And, while there are no concrete predictions based directly on these posits, it is clear that the above explanations disappear without zymosis. Thus, while zymotic material might not have been primarily responsible for the theory's predictive success, it was certainly crucially implicated in the theory's explanatory success. Without the fermentation aspect of zymosis, the entire disease mechanism would have disappeared, and with it disease pathology. Moreover, without it, the link between diseases and decomposition would have been lost, and, as we have seen, without decomposition, the predictions of the zymotic theory disap-

\footnotetext{
${ }^{4}$ Peters (2014) stresses the importance of this in accounts of essentialness. For this reason, the zymotic theory might also turn out to be problematic for Peters' proposal. However, due to space constraints, I will not pursue this further here.
} 
pear, too 5

\section{Conclusion}

What, then, is the upshot of this? What is clear is that the zymotic theory made use-novel predictions, such as those about air quality, and Farr's predictions about elevation and density. Miasmatic posits were essential derivation-internal working posits that, further, could not be weakened, and, so, according to Vickers, they deserved realist commitment. Yet, they were rejected, and no miasmatic posits, as we have seen, were carried over to the germ theory. Zymotic posits were not involved in the predictions in these crucial ways, but were essential to the theory's explanatory power, since, taking away zymosis left a disease theory without a disease mechanism, without a pathology, and without any explanation of many of the well-known disease phenomena that needed accounting for, including the all-important link to decomposition. Thus, even modern, sophisticated selective realist accounts, such as that of Vickers, cannot rise to the historical challenge that underlies the pessimistic meta-induction. Realism remains in as much trouble as ever, at least on this front.

\section{References}

Eyler, John M. (1979). Victorian social medicine: the ideas and methods of William Farr. Johns Hopkins University Press.

Eyler, John M. (1980). "The Conversion of Angus Smith". Bulletin of the History of Medicine, 54(2), 216.

Farr, William (1842). "Letter", 4th Annual Report to the Registrar General, Appendix, London: W. Clowes

Farr, William. (1852). Report on the Mortality of Cholera in England, 184849, London: W. Clowes.

\footnotetext{
${ }^{5}$ For brevity, I cannot discuss in detail why zymes cannot be regarded as proto-germs. I want to stress, however, that even if such an argument could be made (although I am confident that it couldn't), this does not help the realist, since the miasmatic posits were the ones responsible for the zymotic theory's novel predictions, and miasma certainly did not get retained in the germ theory.
} 
Frost-Arnold, Greg (2014). "Can the Pessimistic Induction be Saved from Semantic Anti-Realism about Scientific Theory?" British Journal for the Philosophy of Science 65(3): 521-548.

Harker, David (2013). "How to Split a Theory: Defending Selective Realism and Convergence without Proximity". British Journal for the Philosophy of Science, 64(1): 79-106.

Kitcher, Philip (1993). The Advancement of Science. Oxford University Press

Lyons, Timothy D. (2006). "Scientific realism and the stratagema de divide et impera". British Journal for the Philosophy of Science 57(3):537-560.

Peters, Dean (2014). "What Elements of Successful Scientific Theories Are the Correct Targets for "Selective" Scientific Realism?". Philosophy of Science 81(3): 377-397.

Psillos, Stathis (1999). Scientific Realism: How Science Tracks Truth. Routledge

Saatsi, Juha (2005). "Reconsidering the Fresnel-Maxwell Case Study". Studies in History and Philosophy of Science, 36: 50-38.

Smith, Robert A. (1848). "On the air and water of towns", Report B.A.A.S. 18: $16-31$.

Smith, Robert A. (1859). "On the air of towns". Q. J. Chem. Soc. Lond.. 11: 196-235.

Vickers, Peter (2013). "A Confrontation of Convergent Realism". Philosophy of Science 80(2): 189-211.

Worrall, John (1989). "Structural realism: The best of both worlds?" $D i$ alectica, 43(1-2): 99-124. 